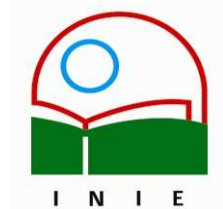

Actualidades Investigativas en Educación

Revista Electrónica publicada por el

Instituto de Investigación en Educación

Universidad de Costa Rica

ISSN 1409-4703

http://revista.inie.ucr.ac.cr

COSTA RICA

TASAS DE DESERCIÓN EN LA UNIVERSIDAD ESTATAL A DISTANCIA DE COSTA RICA

DROP-OUTS FROM THE UNIVERSIDAD ESTATAL A

DISTANCIA DE COSTA RICA

Volumen 8, Número 1

pp. 1-32

Este número se publicó el 30 de abril 2008

Mario Castillo Sánchez

La revista está indexada en los directorios:

LATINDEX, REDALYC, IRESIE, CLASE, DIALNET, DOAJ, E-REVIST@S,

La revista está incluida en los sitios:

REDIE, RINACE, OEI, MAESTROTECA, HUASCARAN

Los contenidos de este artículo están bajo una licencia Creative Commons

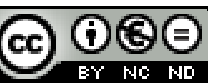




\title{
TASAS DE DESERCIÓN EN LA UNIVERSIDAD ESTATAL A DISTANCIA DE COSTA RICA \\ DROP-OUTS FROM THE UNIVERSIDAD ESTATAL A \\ DISTANCIA DE COSTA RICA
}

\begin{abstract}
Mario Castillo Sánchez ${ }^{1}$
Resumen: La pretensión fundamental del presente trabajo es contribuir al conocimiento de la deserción estudiantil en la UNED; si bien es cierto, la deserción afecta directamente al estudiante, también es un síntoma de fallos en el sistema.

El cambio que conlleva el ingresar a una universidad no es fácil y sobre todo cuando es a distancia, se observa en los resultados que los primeros años es la etapa más difícil de superar, es así como en los primeros 6 meses el 35\% de los estudiantes han hecho abandono de sus estudios. A los 12 meses el $50 \%$ de los estudiantes han abandonado la UNED. En cuanto al género, se observa que las mujeres mantienen una menor tasa de deserción a lo largo de todo el periodo, sin embargo, independientemente del género las tasas alcanzadas son altas.

En cuanto al hecho de tener pareja, pareciera que es un factor que determina un incremento en las tasas de deserción. En relación a los estudios previos, se observa como los estudiantes que han tenido una experiencia universitaria completa, tienen tasas altas de abandono, un $79 \%$ a los 36 meses.

Otro factor importante en las tasas de abandono es el número de materias matriculadas, los estudiantes que matriculan 4 materias o más presentan tasas altas de abandono del 68\% a los 36 meses. La edad parece indicar que a mayor edad mayor tasa de abandono, así los estudiantes con más de 30 años alcanzan una tasa de abandono de $74 \%$ a los 36 meses.
\end{abstract}

\section{Palabras claves: TASAS DE DESERCIÓN, TABLAS DE VIDA, DESERCIÓN UNIVERSITARIA}

\begin{abstract}
The aim of this study is to contribute to the knowledge of the frequent desertion of students in one of the four public universities of Costa Rica, the UNED. Although, it is true that desertion affects directly to students, it is caused by mistakes in the system.

The impact that brings with it the change from high school to college is not easy, and even more if the dynamic of the college is not the usual-distance learning. Our results show that the first years of adaptation are the most difficult ones for the students. During the first 6 months, the 35\% of the students have dropped their classes; and by the end of the 12 months the $50 \%$ of the students have quitted the university. About gender, we can see that females maintain a small percentage of dropping the university than males, however, the numbers are still significantly high. For those students who live with their sentimental companion, this fact seems to significantly affect their decision of dropping the university.

Another import factor in the desertion of students in this university is the number of classes that students register each semester. Students who registered 4 classes or more have a rate of $68 \%$ of desertion at the 36 months. Age seems to indicate to be another important factor-older the student, highest the probability of desertion. Students 30 years old or older reach the $74 \%$ of students who dropped the university at the 36 months of been there.
\end{abstract}

Key Word: RATES DROPOUT, LIFE TABLES, DROPOUT UNIVERSITY

\footnotetext{
${ }^{1}$ Doctor en Educación por la Universidad Estatal a Distancia. Máster en Estadística por la Universidad de Costa Rica. Egresado de la Licenciatura en la Enseñanza de la Matemática por la Universidad Nacional. Profesor de la Escuela de Matemática de la UNA, Escuela de Salud Pública de la UCR y del Programa de Doctorado en Educación de la UNED. Actualmente Subdirector de la Escuela de Matemática de la UNA.
}

Direcciones electrónicas: mcastill@una.ac.cr, mariocastillosp@gmail.com

Artículo recibido: 16 de octubre, 2007

Aprobado: 15 de abril, 2008 


\section{Introducción}

Una situación que se presenta en todas las instituciones educativas y que siempre ha llamado a la reflexión es el abandono de los estudios, pero cuando éste alcanza niveles no manejables se convierte realmente en un gran problema social e institucional.

Este tema siempre ha preocupado a los educadores, sin embargo, la mayoría de las investigaciones en este campo, llegan solamente a una descripción de la situación, sin ahondar en el asunto, y la mayoría de estos estudios se enfoca a la educación primaria o secundaria. A nivel universitario casi siempre se reportan porcentajes brutos de deserción como resultado de estudios de investigación transversal.

Específicamente en el campo de la educación a distancia se han llevado a cabo investigaciones para determinar los niveles de deserción, Universidades como la Universidad Alemana de Leipzig mantuvo una tasa de deserción relativamente alta, igualmente algunas universidades norteamericanas han reportado tasas de deserción anuales de $20 \%$ a $40 \%$ (Kempfer, 1996).

En Estados Unidos se realiza en el año 1999 una investigación, por parte de Adelman citado por Giovagnoli (2002) utilizando datos del Centro Nacional de Estadísticas Educativas, para identificar los factores que contribuyen a que un alumno se gradúe y encuentra que los recursos académicos y la asistencia a clases son los factores que más se correlacionan con la graduación; en el 2001 DesJardin, citado por Giovagnoli (2002), utilizando los mismos datos, determina que el promedio de ingreso es el mejor predictor para la culminación de los estudios con éxito.

En el ámbito latinoamericano e igualmente en el ámbito de la educación a distancia, especialmente en algunos países de América del Sur, se han realizado investigaciones sobre la deserción durante el primer año universitario de los estudiantes de primer ingreso, es así como en Argentina se reportan tasas de deserción del 43\% para los jóvenes universitarios, en este sentido la Lic. Marta Teobaldo (2004) realizó un estudio con el propósito de exponer las razones por las cuales abandonan los estudios los estudiantes de primer año del Ciclo Básico Común de la Universidad de Buenos Aires, un hallazgo interesante es que los estudiantes indican que el factor económico no es determinante en la decisión de continuar en la universidad; la autora considera que es necesario aprender el 
oficio de estudiante universitario, para mantenerse en la universidad, es decir, el estudiante tiene que adaptarse a nuevos estilos y modelos de docentes, diferentes normativas y en general al funcionamiento de la institución.

En Perú se reportan tasas de deserción universitarias superiores al 40\%, y específicamente en el área de la Salud en los estudiantes de Enfermería del $75 \%$, en este punto Hernán Sanabria (2002) de la Universidad Nacional de San Marcos, realizó en el año 2002 un estudio en estudiantes de Enfermería en cuatro universidades del Perú, y concluyó que existe una alta asociación entre deserción y los factores vocacional y económico, así como una leve a moderada asociación del factor rendimiento con la deserción.

En el caso de Brasil, Montoya Díaz en 1999, citado por Giovagnoli (2002) concluye que el ingreso familiar es un factor determinante a la hora de continuar estudios universitarios, es decir que aquellos con un ingreso familiar alto tienen mayor probabilidad de obtener el título universitario que aquellos con ingresos familiares bajos.

En la mayoría de las investigaciones realizadas sobre la deserción de los estudiantes universitarios del sistema a distancia, se enmarcan en dos situaciones particulares del estudiante, por un lado, la integración al ambiente académico y social, indicando que a mayor grado de integración corresponde un mayor grado de compromiso institucional y por otro lado la situación de desgaste del estudiante, atribuyendo la decisión de desertar a factores externos a la institución. Cabrera (1993), citado por Giovagnoli (2002), considera que los factores institucionales y personales del individuo se complementan a la hora de tomar la decisión de abandonar la universidad.

La pretensión fundamental del presente artículo es contribuir al conocimiento de la deserción estudiantil en la Universidad Estatal a Distancia, si bien es cierto la deserción afecta directamente al estudiante, también es un síntoma de fallos en el sistema universitario, es aquí donde radica la importancia de conocer la magnitud de la deserción en la Universidad, pero no solamente la tasa bruta de deserción sino conocer la magnitud de las tasas específicas de deserción.

El abandono de los estudios es un fenómeno que afecta a todas las universidades, sin embargo, el incremento a niveles no manejables, la hacen merecedora de un interés 
primordial dentro del conjunto de problemas que existen en el ámbito universitario. Es evidente la pérdida de recursos económicos y su efecto negativo sobre la necesidad de formación creciente de capital humano, así como el desaliento y el sentimiento de frustración que produce en los estudiantes. Sin embargo, a pesar de su importancia, se han realizado muy pocos estudios para apreciar la magnitud del problema, sus causas y las posibles medidas para enfrentarlo.

En el caso de la Universidad Estatal a Distancia, a este respecto no existen datos básicos suficientemente detallados sobre el progreso de los estudiantes y la deserción. Por esta razón, se ha considerado necesario investigar las tasas de deserción, así como las características relacionadas con esta situación, tanto para el desarrollo de estrategias que disminuyan el problema, como también para considerarlas en la planificación del desarrollo académico de la institución.

Las múltiples consecuencias de la deserción no afectan solamente al individuo, sino que competen a la sociedad en general. La universidad es responsable de formar los recursos humanos necesarios para el desarrollo nacional, de transmitir el patrimonio cultural, constituir el eje del sistema científico tecnológico, difundir la cultura, realizar una labor de crítica social y contribuir al desarrollo del país.

Los costos de la educación son muy altos y la inversión que se hace en este campo debe recuperarse a través de la formación de un tipo de ciudadano cada vez más apto y capaz, por esto la deserción estudiantil debe ser objeto de mayor preocupación e interés, ya que sus implicaciones para la realización de los objetivos de la universidad son de suma importancia.

Las causas de la deserción del estudiante podrían estar determinadas por condiciones objetivas, particularmente características socioeconómicas, psicológicas, culturales propias del estudiante y del entorno en el cual se desenvuelve; así como factores propios de la institución y del sistema de enseñanza y aprendizaje que, de manera directa o indirecta, interactúan favoreciendo que se produzca la deserción. Es aquí donde el término deserción adquiere un sentido sociológico muy amplio. 


\section{Referente teórico}

\subsection{El sistema universitario costarricense}

La historia del sistema universitario costarricense no inicia con la creación de la Universidad de Costa Rica, que se realiza en 1940, no obstante a partir de ese momento, la educación tuvo un impulso que sería de gran importancia en las décadas posteriores.

Las instituciones de la sociedad costarricense, fundamentalmente de servicios, necesitaban técnicos y profesionales en las más diversas áreas. Por esta razón el gobierno se vio en la necesidad de crear nuevos centros de educación superior: entre 1971 y 1978 se crean las nuevas universidades: primeramente se creó el Instituto Tecnológico de Costa Rica (ITCR) en 1971, después la Universidad Nacional (UNA) en el año 1973 y finalmente en el año 1978 la Universidad Estatal a Distancia (UNED). En este mismo periodo se creó la primera universidad privada del país: la Universidad Autonóma de Centroamérica (UACA) en el año 1975 (Vargas, 1996).

Posteriormente, en los ochenta y a inicios de los noventa, se presenta un incremento extraordinario de universidades privadas, crecimiento que se continúa dando hasta la fecha (Jofré, 1998).

\subsection{Situación actual y tendencias de la educación superior}

Una de las situaciones que tuvo y que tiene que enfrentar la educación superior costarricense fue el crecimiento demográfico de los años cincuenta. A partir de 1967 se inicia la incorporación masiva de estudiantes a la universidad, con altas tasas anuales de crecimiento. Costa Rica pasó por altos períodos de crecimiento demográfico en los años 50 e inicios de los 60 , lo que vino a afectar la población en edad de entrada a las universidades de 1967 a 1981; se incrementó más de siete veces la cantidad de estudiantes en las universidades costarricenses, pasando de 6000 a 54000, crecimiento que significó un incremento del $790 \%$ y distorsionó totalmente las tendencias históricas (Jofré, 1998).

El "baby boom" de 1955 a 1965 implica que como promedio, anualmente se estén incorporando, desde 1976 (al llegar a una edad de 21 años) más de 56000 ciudadanos a la vida laboral o a la educación superior (UNED, 1984).

Es importante indicar que algunos investigadores consignan un nuevo boom demográfico entre 1975 y 1985, esta población ejercerá presión en los años venideros por un cupo en la 
educación superior y por ende una mayor calidad de educación; es en este aspecto donde se debe dar la competencia entre universidades públicas y privadas (Ruiz, 2001).

Desde la instauración del sistema educativo superior, la consecución de un título universitario se transformó en un gran reto para la familia costarricense y desde el punto de vista social, se gestó un mecanismo de promoción social o movilidad social que tendría consecuencias para el desarrollo equilibrado de la sociedad.

Este desafío por alcanzar un título universitario, llega también al sector femenino, produciéndose un fenómeno de suma importancia para nuestro país: la incorporación de la mujer al sistema educativo universitario, que se inicia a partir de los años setenta (Jofré, 1998).

Costa Rica no escapa a la crisis económica que ha tenido prácticamente toda la humanidad y ya para la década de los ochenta se da una disminución en el presupuesto destinado para educación en general en el país. Si bien es cierto que según este indicador Costa Rica aún se mantiene en un sitio de privilegio en el área latinoamericana, también es cierto que ha perdido terreno y que presenta una tendencia hacia la declinación (Jofré, 1998).

El mundo se dirige hacia la globalización en muchos sentidos, pero serán las comunicaciones y la penetración masiva de productos y servicios, las que con más fuerza modificarán valores, modos de vida y costumbres. Se sostiene que la tendencia será hacia una pérdida cada vez mayor de las diferencias culturales (Ruiz, 2001).

Jofré (1998) argumenta que, el mundo camina hacia la estandarización, hacia la imposición de valores de sociedades más industrializadas, hacia la universalización de los valores. El desarrollo de los medios de comunicación, la globalización de los mercados, la pérdida de relevancia de las fronteras, irán acabando pausadamente las identidades culturales. Lo que antes llevaba siglos, ahora se produce en unas pocas décadas.

En cuanto a este tema, la universidad no solo tiene la gran oportunidad de establecer el engranaje necesario para rescatar las diferencias culturales y transformar esto en una orientación que desvíe en algún modo la tendencia estandarizadora, sino que tiene también 
la ocasión de recuperar su papel en la sociedad y dejar de verse como una torre de marfil (Jofré, 1998).

En este sentido, la universidad tiene que ser protagonista, fundamentada en su misión de conservar y transmitir la cultura; la universidad no puede mostrarse indiferente en relación con las costumbres, ideas, expresiones artísticas y los valores que han fortalecido a la sociedad.

En este aspecto, Ángel Ruiz (2001) considera que como efecto de la globalización, la educación en general y sobre todo la educación superior, tiene que mejorar la calidad de la educación, no solo en la formación de sus graduados, sino también en un manejo eficaz de los recursos con que cuenta, así como contextualizar sus carreras dando énfasis al tiempo destinado para graduarse, al manejo eficaz de sus recursos, a la pertinencia del currículo, y a la calidad y formación de sus docentes.

Un fenómeno interesante y que cabe mencionar, es que el nuevo boom demográfico cambiará las condiciones económicas del país en las primeras dos décadas del presente siglo, según el Dr. Rosero, la población del primer boom no está aún vieja, trabaja produce y ahorra y por otro lado la generación del segundo boom se está incorporando a la producción del país, por lo que tiende a disminuir el número de dependientes por familia. Sin embargo, para el año 2020 la población del primer boom estará ya en la tercera edad y se terminará esta etapa favorable económicamente para el país. Esto indica que estos primeros años de siglo serán determinantes para el progreso del país. $Y$ es aquí donde las universidades públicas y privadas tienen una gran responsabilidad aunque no exclusiva, para formar esta nueva generación que ha vivido tiempos difíciles económicos y de estilo de vida (Ruiz, 2001).

\subsection{Educación a Distancia en América Latina}

En el contexto de la Educación a Distancia es importante hacer una breve reseña histórica, para analizar sus raíces y la evolución que ha tenido a través de los años, por tal razón se analiza en este apartado la historia de la Educación a Distancia a nivel mundial; posteriormente se investiga propiamente la situación de América Latina, para concluir con la Educación a Distancia en Costa Rica (UNED) y sus perspectivas actuales. 


\subsubsection{Reseña histórica de la Educación a Distancia}

Si bien es cierto que con certeza no se conoce el inicio de la Educación a Distancia, varias investigaciones lo remontan a los siglos XVIII y XIX con las primeras experiencias de enseñanza por correspondencia. Algunos autores van aún más allá para buscar los comienzos de la enseñanza por correspondencia en la historia de la escritura, el desarrollo del alfabeto y el arte de la lectura.

Recientes descubrimientos han permitido concluir que el estudio a distancia organizado se remonta al siglo XVIII, con un anuncio publicado en 1728 por la gaceta de Boston, en donde se hace referencia a un material auto-instructivo para ser enviado a los estudiantes con posibilidad de tutoría por correspondencia. Posteriormente en 1833, en un periódico sueco se anunciaba explícitamente este tipo de enseñanza (Barrantes, 1992).

En 1840 Isaac Pitman organizó en Inglaterra un intento rudimentario de educación por correspondencia. La educación por correspondencia ha servido de base a las primeras posibilidades que se han materializado en el campo de la educación a distancia.

En la Europa Occidental y América del Norte, la educación a distancia empezó en las ciudades industriales del siglo XIX, con el fin de atender a las minorías que no podían asistir a la escuela tradicional (Barrantes, 1992).

Ya en este siglo, en las décadas de los sesenta y setenta, se ha dado un crecimiento explosivo de la educación a distancia en todo el mundo. Es así como entre 1960 y 1975 se fundaron en África más de veinte instituciones de educación a distancia. Entre 1972 y 1980, en Australia, el número de instituciones a distancia pasó de 15 a 48 (Barrantes, 1992).

En Latinoamérica una gran cantidad de países cuenta con algún tipo de sistema de educación a distancia, tema que se analiza en el siguiente apartado.

\subsubsection{El Caso de América Latina}

En América Latina, los sistemas de educación a distancia se han visto como un afán por superar el subdesarrollo, al percatarse que la educación constituye un factor importante para alcanzar el desarrollo. 
Como respuesta a las demandas en educación, los gobiernos latinoamericanos comenzaron a experimentar con nuevas formas de instrucción, en un afán por renovar las estructuras y técnicas educativas (Escotet, 1980).

Es así como nace una de las primeras instituciones de educación superior en América Latina que utiliza un sistema abierto, el Instituto Tecnológico de Estudios Superiores de Monterrey, México. En este país, la Universidad Nacional Autónoma de México, también ha sido pionera en esta modalidad de instrucción (Escotet, 1980).

En la zona de los Andes se destacan la Universidad Nacional Abierta de Venezuela Fundada en 1977 y puesta en operación en 1978. También existen otras experiencias importantes entre las que destacan, los Estudios Libres de la Universidad Simón Bolívar, los Estudios Supervisados en Educación de la Universidad Central de Venezuela, los Estudios a Distancia de la Universidad Simón Rodríguez y los estudios Supervisados de la Universidad del Zulia (Escotet, 1980).

Colombia por su parte, cuenta con varias experiencias de educación a distancia siendo quizás la Universidad de Antioquía y la Universidad Javeriana pioneras en este campo.

En Perú se destaca el Centro de Teleducación de la Universidad Católica. En Ecuador existe un proyecto bilingüe de las Escuelas Radiofónicas de los Centros Shuar, dirigido a dicho grupo cultural en el nivel primario y en Bolivia la Universidad Gabriel René Moreno tiene una audencia no sistematizada de cerca de 40000 personas en Educación para el hogar, salud higiene y saneamiento ambiental (Escotet, 1980).

Argentina, no escapa a este auge de la educación a distancia y la Universidad de Buenos Aires cuenta desde hace tiempo con estudios libres que utilizan esta modalidad de educación.

En Centroamérica y el Caribe, merece especial mención la Universidad Estatal a Distancia de Costa Rica. Otras experiencias importantes son el de instrucción por radio de El Salvador (1978) y la enseñanza de la matemática por Radio de Nicaragua (1978). 
En general, se puede apreciar la existencia de toda una gran diversidad de proyectos y programas en la educación a distancia Latinoamericana.

\subsubsection{La Educación a Distancia en Costa Rica, UNED}

Además de la Universidad De Costa Rica, surge en los años setenta una nueva institución de educación superior, el Instituto Tecnológico de Costa Rica con el fin de capacitar técnicos de nivel medio, directamente en función de las necesidades del desarrollo económico y en especial, del desarrollo de la industria. La Universidad Nacional, surge bajo el influjo directo de las fuerzas de izquierda del momento. De hecho se configura, desde su misma génesis, como una universidad fuertemente politizada e ideologizada que, inclusive, tiende a radicalizar la opción critica y contestaria de la UCR (González, 1984).

La UNED nace poco después, en 1977 y surge con una misión democratizante de la educación superior, orientada hacia la apertura de opciones de educación superior accesibles a los más amplios sectores de la población. $Y$ en particular, accesible para quienes, por razones económicas, laborales, geográficas o familiares, no les es factible asistir a los sistemas universitarios tradicionales (Vargas, 1996).

La educación a distancia, es reconocida como una modalidad destinada a una población geográficamente dispersa, lo cual significa que puede interactuar con un gran número de personas, sin la necesidad de que todas ellas compartan un mismo lugar o recinto físico al mismo tiempo. Así mismo, se basa en el uso intensivo de las nuevas tecnologías de comunicación, audio, video y computadores.

En la Educación a Distancia cambia radicalmente el concepto de enseñanza aprendizaje que se tiene en la modalidad presencial. De un proceso centrado en el que enseña, se produce un viraje que coloca al estudiante como el sujeto protagónico de su propio aprendizaje.

La UNED se especializa en la metodología de enseñanza a distancia, donde se utiliza como principal medio la "unidad didáctica modular" que consiste en un conjunto de recursos didácticos confeccionados y dirigidos a la consecución del autoaprendizaje del estudiante. Esta metodología modifica esencialmente el papel del profesor y transfiere gran responsabilidad al estudiante, pues en este sistema el educando puede laborar y cumplir con 
sus obligaciones familiares porque la metodología a distancia no establece horarios rígidos como en el sistema tradicional.

Se concibe así una institución que funcionará a partir de una metodología novedosa- la de la educación a distancia- y surge con el fin de dar cumplimiento a los siguientes objetivos:

-Atender eficazmente y a más bajo costo las altas demandas educativas que sobrepasan la capacidad instalada, física, académica y económica del sistema tradicional.

-Educar la población adulta, laboral y dispersa.

-Brindar educación permanente.

-Difundir la cultura sin distingos de ningún tipo.

-Provocar un efecto multiplicador de la educación y la cultura.

-Reducir al mínimo las necesidades de planta física y recursos humanos.

-Eliminar el sujeto educando dependiente y crear al autoregulador de su aprendizaje.

- Llevar la educación y la cultura en forma efectiva a todo el territorio. (González,1984)

\subsubsection{La UNED y sus perspectivas hacia el siglo XXI}

Según proyecciones de la UNESCO hacia el 2000, a lo sumo el 10\% de la población de los países en desarrollo estará matriculada en la educación superior, por lo que la universidad tiene el gran desafío de lograr una mayor equidad en el acceso sin que esto repercuta en la calidad (Finardi,1997).

En el presente siglo no se podría hablar de una educación universal, de un modelo único, sino que hay que hablar de modelos educativos que respondan a diferentes grupos sociales, diferentes también en sus niveles de desarrollo y en sus características culturales.

El conocimiento, o mejor dicho la información adquiere cada vez más importancia y es más necesaria en relación con la materia prima, el trabajo y otros recursos. El conocimiento es la nueva ventaja comparativa de las naciones. El factor que decidirá el destino de las naciones en la economía global será el nivel educativo de sus poblaciones. Los nuevos empleos exigen conocimientos, imaginación e inteligencia (Carosio, 1997). 
Por tal razón es insuficiente que los sistemas de educación a distancia brinden solamente los conocimientos técnicos productivos, deben promover conjuntamente con el conocimiento técnico, el desarrollo de la inteligencia y la creatividad (Finardi, 1997).

Otro fenómeno que influirá en los sistemas de educación superior es la creciente participación de la mujer, que podría requerir un cambio en las estructuras actuales y una actualización de los currículos, igualmente los sectores de trabajadores adultos influirán en el currículum pues deberán recalificar sus competencias laborales.

Sin embargo, no podemos obviar que, con las nuevas tecnologías la forma de ver el mundo varía rápidamente: aparecen nuevas representaciones mentales, nuevas experiencias vivenciales, acceso a una cantidad inmensa de información y nuevos entornos de aprendizaje.

La universidad debe preparar al hombre del nuevo siglo, para que sea capaz de asimilar este bombardeo de cambios tecnológicos. No se trata de formar un estudiante receptor, consumidor de información, sino que todo lo contrario, un estudiante activo y reflexivo, por tanto, un constructor de su propio conocimiento.

La Universidad a Distancia se constituye, hoy más que nunca, en un modelo educativo indispensable para nuestra sociedad, que demanda una preparación de diferentes cuadros profesionales, diferenciados por su presenciabilidad en el tiempo y en el espacio y sin que pierdan los niveles de producción socioeconómicamente alcanzados (Corrales, 1997).

Con la globalización, la base de acción son los requerimientos del mercado y la eficiencia productiva es determinante para poder competir a nivel internacional. Esta situación envuelve grandes conflictos y uno de ellos es la repercusión sobre el mercado laboral.

Estos desafíos establecen la necesidad de hacer ajustes en las instancias educativas, para que los trabajadores estén en condiciones de incorporarse adecuadamente al mercado laboral. Esto no es sinónimo de hacer robots perfectamente adaptables a dichos mercados. Es en este aspecto, que el reto de la educación se hace más difícil, porque el sistema educativo debe ser lo suficientemente flexible para el cambio, pero lo adecuadamente 
fundamentado y consolidado como para permitir la formación amplia y crítica de sus estudiantes (Cruz, 1997).

En este sentido, la universidad debe tener presente que la educación debe ser continua, que todo estudiante aún después de graduado deberá regresar a los recintos universitarios para actualizarse.

Así pues, la UNED debe afrontar el desafío que una sociedad en cambio, exige de la educación costarricense, debe por su naturaleza, ser más flexible para ejercer de la mejor manera posible la responsabilidad que comparte con las otras universidades estatales, con las que debe fortalecer y agilizar los más adecuados mecanismos de una permanente coordinación.

\subsection{Conceptualización de la deserción}

La deserción educativa se entiende como un fenómeno que se caracteriza por el abandono definitivo de un estudiante a la institución educativa donde se desempeña. (Villalobos, 1994). En forma general se refiere a una manifestación de conducta, que podría resultar de la interacción de una serie de características o variables, que influyen en la decisión por parte del educando, en este caso específico de un estudiante universitario, por interrumpir sus actividades académicas (Otero, 1983).

Es el resultado de una combinación de factores, e implica un costo social importante, en el sentido de que se da una inversión durante un tiempo determinado, para formar a un individuo, que no culmina su carrera y que por lo tanto no logra los objetivos que en un inicio se había planteado.

La deserción se puede considerar como un parámetro primordial para medir el desarrollo educativo de la población y la eficiencia del sistema educativo de un país, siendo en la actualidad un problema al que se enfrentan miles de estudiantes.

A veces resulta difícil determinar cuándo debe o puede considerarse que un estudiante inscrito ha abandonado sus estudios, en un curso o en una carrera. La situación se torna aún más compleja cuando los alumnos que abandonan sus estudios pueden en cualquier momento volver a la institución universitaria, como ocurre con frecuencia en la educación a distancia. Es decir, dejan uno o más periodos académicos sin estudiar y luego vuelven. Esta 
complicación dificulta mucho la evaluación e interpretación de las cifras sobre este tema, no permitiendo en múltiples oportunidades, comparaciones o conclusiones sólidas rigurosas.

Desde una perspectiva psicosocial, la deserción genera un fuerte impacto en el sujeto que hace abandono de sus estudios. Por ejemplo, produce sentimientos de frustración, que con el paso del tiempo pueden conducir a un sentimiento de fracaso.

Generalmente, cuando las personas encuentran una serie de limitaciones económicas para adquirir los bienes y servicios que desean, asumen como causa de tal hecho la carencia de una preparación académica.

\subsection{Deserción en la UNED}

Desde inicios de la UNED y considerando el sistema novedoso de la educación a distancia en el país, se ha determinado que un problema importante debe ser la deserción estudiantil. Es así como se inicia en 1979 el primer intento de analizar este fenómeno y se aporta la primera cifra al respecto donde se indica que la deserción total fue de 35\%. (Góngora, 1983, citado en Elizondo, 1987). Posteriormente Saborío (1986) señala que en el II semestre de 1984 la deserción fue de un 39.3\% y determina que en el mismo período en 1985 alcanzó un $41,7 \%$. Más tarde, se determina en una muestra de 38 cursos, que la deserción para el II semestre del año 87 alcanzó un 54,59\% (Elizondo, 1987).

Presentadas estas tasas de incidencia de deserción, la UNED ha intentado determinar las causas que inducen a esta decisión. En 1980 y a petición del rector, se realiza un estudio para comprender la situación y definir el problema de la deserción de estudiantes. Más tarde, en 1985, Bolaños realiza un estudio de opinión en 61 desertores de la UNED. Sin embargo, a la fecha no existe una investigación que, utilizando una muestra representativa y técnicas estadísticas adecuadas, determine las causas socioeconómicas, educativas y de apoyo administrativo que aclaren la razón del aumento creciente en los porcentajes de deserción.

No se ha permitido realmente, determinar los diferentes factores que intervienen en el proceso de la deserción y más aún no se han aportado soluciones objetivas con el fin de minimizarlo. Ello se debe a una serie de limitaciones claves presentes en el campo teóricometodológico. 
Además de estas limitaciones, existen otras, que hacen referencia particularmente a la muestra utilizada, a la misma definición operativa de deserción, al procedimiento de muestreo y las fuentes de información utilizadas. La revisión de la literatura realizada hasta el momento, sugiere que en muchos estudios los criterios respecto de la selección de la muestra son poco objetivos y por ende poco confiables. Unido a esto, el estudiante desertor en algunas investigaciones no se ha integrado como fuente de información.

Uno de los propósitos al realizar esta investigación es subsanar las deficiencias metodológicas mencionadas anteriormente, por lo que desde la selección de la muestra hasta el análisis estadístico se ha realizado con el rigor científico que se merece este tema.

\section{Metodología}

La presente investigación se enmarca dentro de los fundamentos del enfoque cuantitativo, según Barrantes (1999, p. 71) "El investigador es un elemento externo al objeto que se investiga. En este proceso utiliza las técnicas estadísticas en el análisis de datos y generaliza los resultados"

Con respecto al diseño es no experimental o ex post-facto, los hechos o variables ya ocurrieron, pues como indica Hernández (1997), se lleva a cabo sin la manipulación deliberada de las variables. Este estudio se ubica dentro los estudios descriptivos, llamados de "desarrollo" los cuales tienen como objetivo investigar patrones y secuencias de desarrollo y/o cambio como una función del tiempo (Colás, 1994).

Según Hernández (1997) la presente investigación es descriptiva y se enmarca dentro del enfoque cuantitativo utilizando un diseño longitudinal de análisis evolutivo de grupo o cohorte, pues lo que interesa es obtener información repetida de los sujetos de estudio a lo largo de varios años, para identificar el proceso y cambios a través del tiempo en subpoblaciones o grupos específicos de interés.

En esta etapa lo que se pretende es determinar el nivel de deserción según características sociodemográficas. En esta parte se utiliza las técnicas de tablas de vida, para determinar las tasas de deserción por cada subgrupo de interés. 


\subsection{Fuentes de Datos}

El estudio de la temática se puede llevar a cabo con los datos del departamento de registro de la Universidad Estatal a Distancia, donde se lleva registro de la matrícula que realizan los estudiantes cada período. En la presente investigación se analizará la cohorte de estudiantes que ingresaron a la UNED en el primer período de 1995. El total de dicha población es de 2560 estudiantes. Debido a la limitación de tiempo y de recursos humanos, se decidió tomar una muestra aleatoria simple de 824 , esto significa que suponiendo un $95 \%$ de confianza y una varianza máxima, el error permisible no sobrepasa el $3 \%$.

Se revisaron los expedientes de todos estos 824 estudiantes para determinar las características de interés.

\subsection{Variables de la investigación}

Las variables a considerar en el estudio, provienen de un cuestionario que tienen que llenar los estudiantes en el momento de hacer la matrícula, para efectos del presente trabajo se determinaron las siguientes variables que corresponden a las características de los estudiantes al inicio del periodo de estudio:

Género: corresponde al sexo de los estudiantes; masculino o femenino

Estado civil: Se determinaron dos subgrupos en pareja y sin pareja

Número de hijos: de establecieron cuatro subgrupos ; 0 hijos, 1 hijo, 2 hijos, 3 y más hijos

Edad de los hijos: se definieron tres subgrupos; no tiene, menos de 6 años y más de 6 años

Estudios previos: dos subgrupos; con estudios universitarios previos y los que no.

Situación Laboral: se determinaron dos grupos los que trabajan y los que no trabajan

Zona de Residencia: dentro de la Gran Área Metropolitana (GAM) y fuera de ella.

Número de materias matriculadas: 1 materia, 2 materias, 3 materias, 4 y más materias.

Edad de los estudiantes: de 17 años a menos de 30 años y 30 años y más.

\subsection{Segmentos de uso}

La unidad de análisis para el estudio de la dinámica de la deserción es el segmento de continuación, que es el período de estudio ininterrumpido de un estudiante. Es decir, al analizar el periodo de 5 años, se considera como desertor al estudiante que en algún momento abandona la universidad y no vuelve a matricular durante estos 5 años. 
Por ejemplo, el primer segmento de continuación para un estudiante, en el archivo corresponde a los meses que van desde el primer período de 1995, hasta la ocurrencia de una deserción en sus estudios.

\subsection{Segmentos no censurados y censurados}

Existen 2 tipos de segmentos de continuación: los completos, que son aquellos cuya duración es conocida porque se tienen sus fechas de inicio y de finalización, y los incompletos o "censurados" que son aquellos de duración indeterminada y de los cuáles sólo se sabe que son mayores que el tiempo de estudio.

En el segundo caso se encuentran los segmentos que empezaron antes del primer periodo de 1995 (censurados por la izquierda) y los que continuaban en la institución al primer periodo del 2000 (censurados por la derecha). Para el presente trabajo se excluirán los segmentos censurados por la izquierda, por la razón de que no existen procedimientos estadísticos para manejar la censura a la izquierda. Esta es la razón de considerar la cohorte de estudiantes de primer ingreso en el periodo 1-95.

\subsection{Tabla de Vida}

La tabla de vida referida muchas veces como tabla de mortalidad es utilizada generalmente por demógrafos para determinar las tasas de mortalidad de una población, y es un instrumento o esquema teórico que permite medir las probabilidades de vida y de muerte de una población, en función de la edad y para un período de tiempo determinado. El concepto original de tabla de vida consiste en seguir una generación o cohorte a lo largo del tiempo, determinando a cada edad el número de sobrevivientes, hasta que la generación se extingue. Las tablas de vida pueden utilizarse para estimar probabilidades en procesos donde interviene el tiempo hasta que ocurre un evento. (Namboodiri, 1987)

La tabla de vida es un procedimiento muy utilizado para el cálculo de tasas de terminación y de interrupción en situaciones de duración-evento, para lo cual se calcula una tasa de terminación para cada mes.

La tabla de vida permite el cálculo de tasas de continuación para cada mes; estas tasas de continuación mensuales pueden ser resumidas para calcular la tasa de continuación acumulada de un año, dos años, o cualquier intervalo de tiempo de interés. 
Para el estudio de las probabilidades de interrupción con las técnicas de la tabla de vida, se debe medir el tiempo continuado de estudio hasta que se produzca la deserción. Sin embargo, muchos estudiantes interrumpen sus estudios únicamente con la intención de posponerlos. Esta situación distorsiona las comparaciones de los niveles de deserción. Para evitar estas complicaciones, se hace el seguimiento exclusivo de las interrupciones consideradas como deserciones, considerando a las otras posibilidades como "censuradas" para el análisis de la deserción.

Para la construcción de las tablas de vida, se utilizó la versión de Potter $(1966,1967)$, recomendaciones hechas por Curtis (1994), y también la información suministrada por Namboodiri (1987). Con el objetivo de calcular las tasas brutas de deserción se utilizó las tablas de vida de decremento único, para tal fin se necesita, la información de los segmentos de permanencia, principalmente, la fecha de inicio y la duración hasta que ocurre la deserción.

Los subgrupos por utilizar en la construcción de las tablas de vida son los indicados anteriormente como categorías de las variables. La nomenclatura por utilizar es la siguiente:

$x$ : $\quad$ unidad de tiempo utilizada.

$N_{x}$ : Intervalos en riesgo en la unidad de tiempo exacta $\mathrm{x}$.

$N^{*}{ }_{x}: \quad$ Intervalos en riesgo corregidos en la unidad de tiempo exacta $\mathrm{x}$.

$S T_{x, x+1}: \quad$ Salidas totales entre los periodos $\mathrm{x}$ y $\mathrm{x}+1$.

$A_{x, x+1}: \quad$ Intervalos censurados.

$q_{x}: \quad \quad \quad$ Probabilidad de salida por todas las causas, en el periodo $\mathrm{x}, \mathrm{x}+1$.

$C_{x+1}$ : $\quad$ Tasa acumulada de continuación en el periodo exacto $\mathrm{x}+1$.

$R_{x+1}$ : $\quad$ Tasa acumulada de salida del período de estudio por todas las causas, en el periodo exacto $\mathrm{x}+1$.

Para efectos de la construcción de las tablas de vida en el programa SPSS se definen dos variables auxiliares: el tiempo y la censura.

Tiempo: Es el tiempo transcurrido (meses) que va desde el primer período de 1995, hasta la ocurrencia de una deserción 
Censura: Es la variable que indica si cada segmento de tiempo se encuentra completo o no. Censura $=0$ segmento censurado (segmento incompleto) Censura $=1$ segmento no censurado (segmento completo)

La construcción de la tabla se desarrolla de la siguiente manera:

1. Los intervalos en riesgo, en el periodo exacto $x$, son:

sin embargo, se $N_{x}=N_{x+1}+S T_{x, x+1}+A_{x, x+1}$ debe considerar la contribución que hacen los segmentos censurados, se supone que ellos son observados a la mitad del periodo, y consecuentemente solo contribuyen con medio periodo de exposición, así, los intervalos en riesgo corregidos son:

$$
N^{*}{ }_{x}=N_{x}-0,5 A_{x, x+1}
$$

2. La probabilidad de desertar para cada periodo, condicional al hecho de que el evento no ha ocurrido en un periodo anterior, se calcula como:

$$
q_{x}=\frac{S T_{x, x+1}}{N_{x}^{*}}
$$

3. La tasa acumulada de continuación en el periodo $x+1$ es

4. La tasa acumulada de abandono es:

$$
C_{x+1}=\left(1-q_{x}\right) \cdot C_{x}
$$

$$
R_{x+1}=1-C_{x+1}
$$

Luego de exponer la metodología utilizada, se procede con el análisis de los resultados.

\section{Resultados}

La deserción al igual que cualquier fenómeno es el resultado de múltiples factores, y es muy difícil poder examinar exhaustivamente todos y cada uno de los elementos que determinan el hecho de por qué un estudiante decide abandonar sus estudios.

En el cuadro № 1 y gráfico № 1 se presentan las tasas acumuladas de abandono a diferentes meses. 


\section{CUADRO 1}

UNED: TASAS ACUMULADAS DE ABANDONO PARA UNA MUESTRA DE ESTUDIANTES INGRESADOS EN 1995 SEGÚN MES, EN EL PERIODO 1995-2000

\begin{tabular}{ccc}
\hline Duración & Tasa Abandono & Error estándar \\
\hline 6 meses & 0.35 & 0.02 \\
12 meses & 0.50 & 0.02 \\
18 meses & 0.56 & 0.02 \\
24 meses & 0.56 & 0.02 \\
30 meses & 0.60 & 0.02 \\
36 meses & 0.62 & 0.02 \\
42 meses & 0.63 & 0.02 \\
48 meses & 0.63 & 0.02 \\
\hline
\end{tabular}

Se destaca que al término de los primeros 6 meses el $35 \%$ ha hecho abandono de sus estudios, es decir que en los primeros 6 meses únicamente el $65 \%$ continua con sus estudios. A los 12 meses el 50\% de los estudiantes han abandonado la UNED, la tendencia de abandono en los siguientes meses no es tan marcada permaneciendo casi constante después de los 42 meses con una tasa de abandono del $63 \%$, estas tasas son mayores a las encontradas por Teobaldo (2004) en Argentina y son mayores a las tasas reportadas por Kempfer (1996) en las universidades de Tailandia y en la Universidad Estatal a Distancia de España.

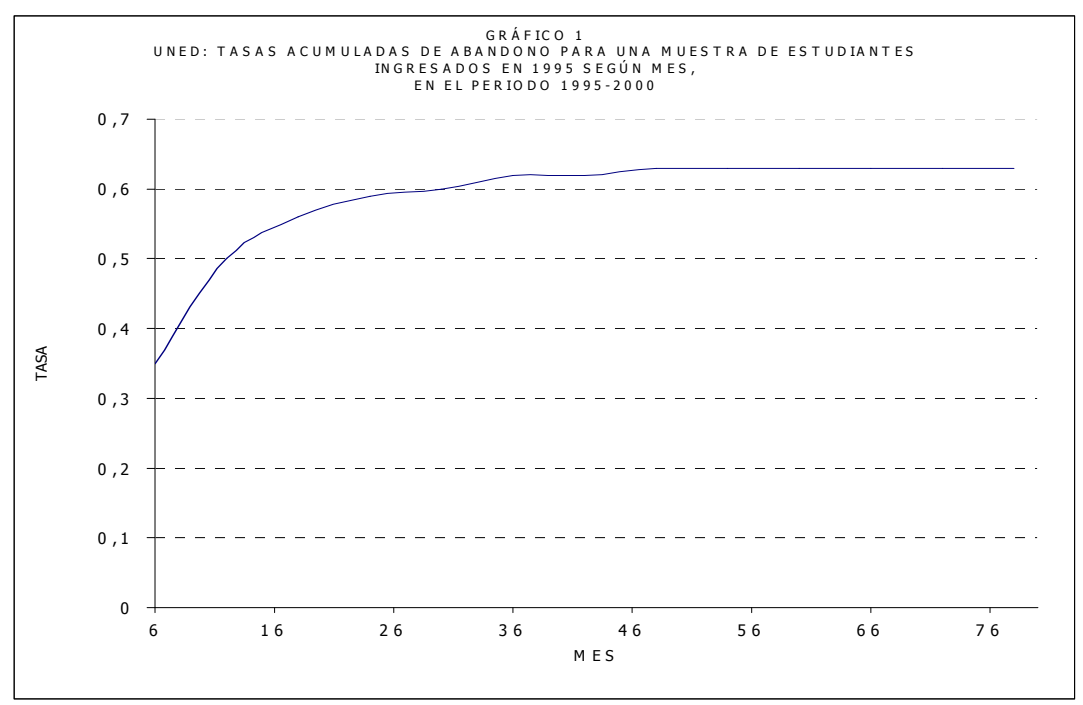




\subsection{Tasas de abandono de los diferentes grupos sociodemográficos}

Género: Al analizar la deserción por género, se observa en el cuadro № 2 y gráfico № 2 , que las mujeres mantienen una menor tasa de deserción a lo largo de todo el periodo de observación, es importante notar aquí que la proporción de mujeres en la muestra es mayor. La tasa de deserción en el grupo de las mujeres no alcanza el 63\% referido anteriormente, llega a un $62 \%$ después del mes $48^{\circ}$, los hombres al contrario alcanzan un $64 \%$ a los 36 meses. Se puede indicar también que al cabo del $48^{\circ}$ mes, únicamente el $38 \%$ de las mujeres y el $36 \%$ de los hombres se mantienen en sus estudios. Se nota como estas tasas independientemente del género del estudiante son realmente altas. Especialmente los hombres tienen tasas mayores durante todo el periodo, este hecho confirma los resultados obtenidos por otros investigadores como García Arieto (1987) en la Universidad Estatal a Distancia en España y Giovagnoli (2002) en Argentina, pero contrasta por lo encontrado por Granados (1992) en España donde determina que el género no causa diferencias significativas a la hora de desertar.

\section{CUADRO 2}

UNED. TASAS ACUMULADAS DE ABANDONO PARA UNA MUESTRA DE ESTUDIANTES INGRESADOS EN 1995 SEGÚN MES POR GÉNERO,

EN EL PERIODO 1995-2000

\begin{tabular}{ccc}
\hline Duración & Tasa Femenina & Tasa Masculina \\
\hline $\mathrm{N}$ & 534 & 290 \\
6 meses & 0.33 & 0.38 \\
12 meses & 0.48 & 0.53 \\
18 meses & 0.54 & 0.60 \\
24 meses & 0.58 & 0.61 \\
30 meses & 0.60 & 0.62 \\
36 meses & 0.61 & 0.64 \\
42 meses & 0.61 & 0.64 \\
48 meses & 0.62 & 0.64 \\
\hline
\end{tabular}




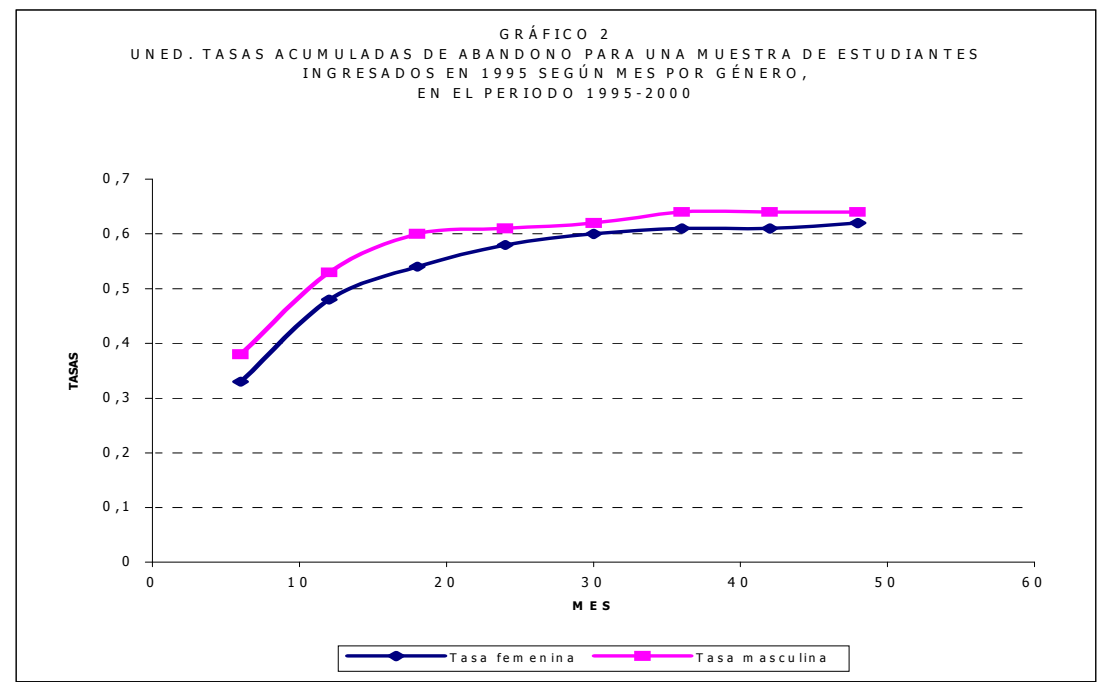

Estado civil: Para hacer el análisis del estado civil, se dividió la muestra, en los estudiantes con pareja y sin pareja, se observa en el cuadro № 3, el hecho de la no diferencia entre estos dos grupos en los primeros meses, pero después del primer año la deserción es mayor en los estudiantes que mantienen una relación de pareja, sin embargo después de 36 meses estos mismos estudiantes mantienen una menor tasa de deserción que los estudiantes solos, alcanzando los niveles de $61 \%$ para los estudiantes sin pareja y de un $59 \%$ para los estudiantes que se encuentran conviviendo con alguien. Este resultado concuerda con los encontrados por García Aretio (1987) en la Universidad Estatal a Distancia de España y con los expuestos por Giovagnoli (2002) en Universidades Argentinas.

\section{CUADRO 3}

UNED. TASAS ACUMULADAS DE ABANDONO PARA UNA MUESTRA DE ESTUDIANTES INGRESADOS EN 1995 SEGÚN MES POR ESTADO CIVIL, EN EL PERIODO 1995-2000

\begin{tabular}{ccc}
\hline & & Estado Civil \\
\cline { 2 - 3 } Duración & Sin Pareja & En Pareja \\
\hline N & 458 & 366 \\
12 meses & 0.35 & 0.35 \\
18 meses & 0.50 & 0.50 \\
24 meses & 0.56 & 0.57 \\
30 meses & 0.59 & 0.59 \\
36 meses & 0.61 & 0.59 \\
42 meses & 0.63 & 0.61 \\
48 meses & 0.63 & 0.61 \\
\hline
\end{tabular}


Número de hijos: Al analizar la deserción por número de hijos el mayor abandono en los estudios se da en el grupo de estudiantes sin hijos a lo largo de todo el periodo, este hecho confirma lo expuesto por García Aretio (1987) quién determinó que en la Universidad Estatal a Distancia de España el grupo que menos deserción tuvo en los estudios era el de estudiantes con hijos, propiamente con 2 o 3 hijos.

Se puede notar en el cuadro № 4 al final del periodo de observación que el $32 \%$ de los estudiantes que no tienen hijos permanece en la Universidad y que el $46 \%$ de los estudiantes que tienen 2 hijos continúan en ella. Los estudiantes que tienen 1 hijo o 3 hijos y más mantienen tasas de deserción muy similares, un $62 \%$ y $63 \%$ respectivamente, y en términos generales se observa que el grupo que presenta las menores tasas de deserción es el grupo con 2 hijos.

\section{CUADRO 4}

UNED. TASAS ACUMULADAS DE ABANDONO PARA UNA MUESTRA DE ESTUDIANTES INGRESADOS EN 1995 SEGÚN MES, POR NÚMERO DE HIJOS, EN EL PERIODO 1995-2000

\begin{tabular}{ccccc}
\hline & & & & Número de hijos \\
\cline { 2 - 5 } Duración & 0 hijos & 1 hijo & 2 hijos & 3 hijos y más \\
\hline $\mathrm{N}$ & 366 & 126 & 207 & 125 \\
6 meses & 0.37 & 0.36 & 0.31 & 0.36 \\
12 meses & 0.53 & 0.50 & 0.44 & 0.51 \\
18 meses & 0.61 & 0.54 & 0.48 & 0.62 \\
24 meses & 0.64 & 0.59 & 0.49 & 0.63 \\
30 meses & 0.66 & 0.60 & 0.50 & 0.63 \\
36 meses & 0.68 & 0.62 & 0.52 & 0.63 \\
42 meses & 0.68 & 0.62 & 0.54 & 0.63 \\
48 meses & 0.68 & 0.62 & 0.54 & 0.63 \\
\hline
\end{tabular}

Edad de los hijos: Es importante analizar la deserción no solo por el número de hijos, sino por la edad de los hijos, este análisis se refiere a la edad del hijo menor. Se observa en el Cuadro № 5 que las menores tasas de deserción se presentan en el grupo donde el hijo menor tiene de 1 a 6 años, igualmente es importante las tasas que se presentan en el grupo con hijos mayores a 6 años, alcanzando un $76 \%$ a los 30 meses, sin embargo durante el primer año no hay diferencias muy marcadas en las tasas de abandono de los estudios. Desafortunadamente no se encontraron trabajos de investigación que consideraran la condición de la edad de los hijos a la hora de tomar la decisión de abandonar los estudios. 


\section{CUADRO 5}

UNED. TASAS ACUMULADAS DE ABANDONO PARA UNA MUESTRA DE ESTUDIANTES INGRESADOS EN 1995 SEGÚN MES, POR EDAD DEL HIJO MENOR EN EL PERIODO 1995-2000

\begin{tabular}{cccc}
\hline & \multicolumn{3}{c}{ Edad del hijo menor } \\
\cline { 2 - 4 } Duración & No tiene & $1-6$ años & 6 años y más \\
\hline $\mathrm{n}$ & 366 & 399 & 59 \\
6 meses & 0.37 & 0.33 & 0.35 \\
12 meses & 0.53 & 0.46 & 0.58 \\
18 meses & 0.61 & 0.52 & 0.62 \\
24 meses & 0.64 & 0.53 & 0.70 \\
30 meses & 0.66 & 0.54 & 0.76 \\
36 meses & 0.69 & 0.55 & 0.76 \\
42 meses & 0.69 & 0.55 & 0.76 \\
48 meses & 0.69 & 0.57 & 0.76 \\
\hline
\end{tabular}

Estudios previos: Al hacer un análisis de los estudios previos, se observa en el cuadro № 6 como los estudiantes que han tenido una experiencia universitaria completa, es decir que ya obtuvieron al menos el título de bachiller y que posiblemente se matriculan en las licenciaturas o maestrías de la UNED, tienen tasas altas de abandono, un $79 \%$ a los 36 meses, aquí surge la hipótesis que al venir del sistema tradicional de enseñanza, los estudiantes no están preparados para la enseñanza a distancia, hecho que se corrobora al observar las tasas de abandono de los estudiantes que no tienen estudios previos, estas tasas se mantienen por debajo de los otros tres grupos durante todo el periodo.

\section{CUADRO 6}

UNED. TASAS ACUMULADAS DE ABANDONO PARA UNA MUESTRA DE ESTUDIANTES INGRESADOS EN 1995 SEGÚN MES, POR ESTUDIOS PREVIOS EN EL PERIODO 1995-2000

\begin{tabular}{ccccc}
\hline \multirow{2}{*}{ Duración } & No tiene & Parauniversitarios & $\begin{array}{c}\text { Universitaria } \\
\text { incompleta }\end{array}$ & $\begin{array}{c}\text { Universitaria } \\
\text { completa }\end{array}$ \\
\cline { 2 - 5 } $\mathrm{N}$ & 419 & 156 & 132 & 117 \\
6 meses & 0.32 & 0.38 & 0.38 & 0.37 \\
12 meses & 0.45 & 0.53 & 0.49 & 0.63 \\
18 meses & 0.50 & 0.62 & 0.54 & 0.72 \\
24 meses & 0.53 & 0.65 & 0.56 & 0.75 \\
30 meses & 0.54 & 0.67 & 0.59 & 0.77 \\
36 meses & 0.54 & 0.71 & 0.61 & 0.79 \\
42 meses & 0.54 & 0.71 & 0.61 & 0.79 \\
48 meses & 0.56 & 0.71 & 0.61 & 0.79 \\
\hline
\end{tabular}

Situación Laboral: Otro elemento influyente en la decisión de continuar o no los estudios es el hecho de trabajar, se observa, en el Cuadro № 7, que durante el primer semestre el hecho 
de trabajar no hace diferencia en las tasas de abandono, a partir del primer año las diferencias son más marcadas alcanzando tasas altas hasta de un 65\% para el grupo de estudiantes trabajadores, a los 36 meses, contra un 56\% del grupo que no labora en forma remunerada. Al término del periodo en estudio, únicamente el $35 \%$ de los estudiantes que trabajan permanece en la Universidad contra el $44 \%$ de los estudiantes que no trabajan. A este respecto, García Aretio ( 1987) y Granados (1992) indican que los estudiantes con obligaciones laborales mantienen tasas mayores de deserción que aquellos estudiantes que solo estudian, hecho que se comparte en esta investigación y concuerdan con los encontrados en las Universidades Argentinas por Giovagnoli (2002).

\section{CUADRO 7}

UNED. TASAS ACUMULADAS DE ABANDONO PARA UNA MUESTRA DE ESTUDIANTES INGRESADOS EN 1995 SEGÚN MES, POR SITUACIÓN LABORAL EN EL PERIODO 1995-2000

\begin{tabular}{ccc}
\hline & & Trabaja \\
\cline { 2 - 3 } Duración & No & Si \\
\hline N & 197 & 627 \\
12 meses & 0.34 & 0.35 \\
18 meses & 0.44 & 0.52 \\
24 meses & 0.49 & 0.58 \\
30 meses & 0.54 & 0.61 \\
36 meses & 0.55 & 0.62 \\
42 meses & 0.56 & 0.64 \\
48 meses & 0.56 & 0.64 \\
\hline
\end{tabular}

Zona de Residencia: En el cuadro № 8 se hace el análisis por zona de residencia, se observa como a lo largo de todo el periodo de estudio las tasas más altas se presentan para los residentes de fuera de la Gran Área Metropolitana. Esta tendencia puede darse por la dificultad de acceso a las oficinas centrales de la UNED y por ende una clara falta de identificación con la universidad. También es conocido que en algunas sedes regionales de la UNED existen tutorías que no pueden brindarse por haber muy pocos estudiantes matriculados, teniendo estos que viajar a los centros universitarios del Área Metropolitana. Dentro de la Gran Área Metropolitana las tasas son medias antes de los 18 meses y a partir de este momento se obtienen tasas altas de deserción durante todo el periodo. Resultados coincidentes con los de Granados (1992) en la Universidad Estatal a Distancia de España, quién encontró que los estudiantes procedentes de zonas rurales obtenían tasas mayores de deserción que los de la zona urbana. 
CUADRO 8

UNED. TASAS ACUMULADAS DE ABANDONO PARA UNA MUESTRA DE ESTUDIANTES INGRESADOS EN 1995 SEGÚN MES, POR RESIDENCIA EN EL PERIODO 1995-2000

\begin{tabular}{ccc}
\hline & \multicolumn{2}{c}{ Residencia } \\
\cline { 2 - 3 } Duración & Fuera de la GAM $^{*}$ & Dentro de la GAM \\
\hline $\mathrm{n}$ & 477 & 347 \\
6 meses & 0.39 & 0.29 \\
12 meses & 0.54 & 0.45 \\
18 meses & 0.59 & 0.53 \\
24 meses & 0.62 & 0.55 \\
30 meses & 0.64 & 0.56 \\
36 meses & 0.65 & 0.58 \\
42 meses & 0.65 & 0.58 \\
48 meses & 0.66 & 0.58 \\
\hline
\end{tabular}

*Gran Área Metropolitana.

Número de materias matriculadas: Otro factor importante en las tasas de abandono es el número de materias matriculadas, se observa en el cuadro № 9 que todas las tasas son altas a partir de los 18 meses, así los estudiantes que matriculan 4 materias o más presentan tasas altas de abandono del $68 \%$ a los 36 meses, mientras que los que matriculan solamente 1 materia la tasa de abandono oscila alrededor de $57 \%$ alcanzando un $61 \%$ a los 48 meses, también considerada como alta. Esta situación hace suponer que muchas veces el estudiante no está preparado para llevar un bloque completo en la UNED o a la inversa que los bloques completos de la UNED no están elaborados para estudiantes con las características que presentan los alumnos de esta institución. Concerniente a esto Bolaños(1985) considera necesario proporcionar información y orientación a los estudiantes sobre la carga académica recomendable de matricular, considerando las situaciones personales; de trabajo, las obligaciones familiares, los estudios previos y todas las situaciones que puedan interactuar con su estudio.

A este respecto, García Aretio (1987) determinó que en la Universidad Estatal a Distancia de España, los estudiantes que matricularon tres o cuatro materias eran los que presentaban mayor incidencia en el abandono, resultado que concuerda con lo hallado en esta investigación. 


\section{CUADRO 9}

UNED. TASAS ACUMULADAS DE ABANDONO PARA UNA MUESTRA DE ESTUDIANTES INGRESADOS EN 1995 SEGÚN MES, POR MATERIAS MATRICULADAS EN EL PERIODO 19952000

\begin{tabular}{ccccc}
\hline & \multicolumn{4}{c}{ Materias Matriculadas } \\
\cline { 2 - 5 } Duración & 1 Materia & 2 Materias & 3 Materias & 4 y más materias \\
\hline $\mathrm{n}$ & 131 & 304 & 227 & 162 \\
6 meses & 0.32 & 0.31 & 0.37 & 0.42 \\
12 meses & 0.46 & 0.48 & 0.53 & 0.53 \\
18 meses & 0.52 & 0.54 & 0.60 & 0.60 \\
24 meses & 0.53 & 0.57 & 0.62 & 0.63 \\
30 meses & 0.54 & 0.59 & 0.63 & 0.66 \\
36 meses & 0.57 & 0.61 & 0.64 & 0.68 \\
42 meses & 0.57 & 0.61 & 0.64 & 0.68 \\
48 meses & 0.61 & 0.61 & 0.64 & 0.68 \\
\hline
\end{tabular}

Edad de los estudiantes: Como se observa en el cuadro № 10, el grupo con edades mayores a 30 años presentan tasas altas a partir del primer año. Los resultados indican que a mayor edad mayor tasa de abandono, así los estudiantes con más de 30 años alcanzan una tasa de abandono de $74 \%$ a los 36 meses, mientras que los menores de 30 años llegan al $60 \%$ a los 48 meses. Visto de otra manera, el $26 \%$ de los estudiantes mayores a 30 años continúan sus estudios en la UNED, contra el $40 \%$ de los estudiantes menores a 30 años y existe una marcada diferencia entre los dos grupos a lo largo de todo el periodo en estudio.

Importante indicar que estos resultados contradicen lo reportado por la mayoría de autores consultados, García Aretio (1987) estableció que los de 30 años a 39 años de edad tenían las tasas más bajas de abandono, por su parte Granados (1992) llegó a conclusiones similares indicando además que probablemente el grado de madurez en los estudiantes era menor en los jóvenes propiciando un rápido abandono de la Universidad. 


\section{CUADRO 10}

UNED. TASAS ACUMULADAS DE ABANDONO PARA UNA MUESTRA DE ESTUDIANTES INGRESADOS EN 1995 SEGÚN MES, POR EDAD EN EL PERIODO 1995-2000

\begin{tabular}{ccc}
\hline & \multicolumn{2}{c}{ Edad } \\
\cline { 2 - 3 } Duración & De 17 años a 30 años & Más de 30 años \\
\hline N & 675 & 149 \\
6 meses & 0.34 & 0.41 \\
12 meses & 0.47 & 0.60 \\
18 meses & 0.54 & 0.67 \\
24 meses & 0.56 & 0.71 \\
30 meses & 0.58 & 0.73 \\
36 meses & 0.59 & 0.74 \\
42 meses & 0.59 & 0.74 \\
48 meses & 0.60 & 0.74 \\
\hline
\end{tabular}

\section{Discusión de resultados}

El cambio que conlleva el ingresar a una universidad no es fácil y sobre todo cuando se trata de una universidad a distancia, se puede notar en los resultados obtenidos que los primeros años o cursos son la etapa más difícil de superar es así como en los primeros 6 meses el $35 \%$ de los estudiantes que han matriculado por primera vez ha hecho abandono de sus estudios, y a los 12 meses el $50 \%$ de los estudiantes han abandonado la UNED. Es en esta etapa inicial donde las autoridades de la UNED deben prestar especial atención a los cursos de orientación y de iniciación a este sistema de educación, nuevo para la gran mayoría de estudiantes.

Otro hecho interesante es que las mujeres mantienen una menor tasa de abandono a lo largo de todo el periodo analizado, este hecho podría estar relacionado con la misma condición de la mujer que está más acostumbrada a llevar varios roles dentro de su hogar y al igual como menciona Garland (1994) los hombres podrían tener otras expectativas diferentes a las educacionales posiblemente económicas o sociales.

Al hacer el análisis del estado civil no se presentan diferencias entre los grupos de estudiantes en pareja y los que no mantienen una relación de pareja

Un elemento importante al analizar las tasas de abandono es el número de hijos, pero lo interesante es que la mayor incidencia se presenta en el grupo de estudiantes sin hijos, los que probablemente son jóvenes que vienen saliendo del colegio y no están acostumbrados a una educación superior donde hay una gran responsabilidad del estudiante. 
Al hacer el análisis por la edad del hijo menor, las menores tasas de deserción se presentan en el grupo donde el hijo menor tiene de 1 a 6 años, igualmente es importante las tasas que se presentan en el grupo con hijos mayores a 6 años, alcanzando un $76 \%$ a los 30 meses. Esto puede deberse a que son personas de mayor edad y probablemente con mayor responsabilidad en sus trabajos $u$ hogares.

Un hecho interesante al considerar la experiencia previa en estudios universitarios, es que los estudiantes que han tenido una experiencia universitaria completa, es decir que ya obtuvieron al menos el título de bachiller tienen la mayor tasa de abandono, mientras que los estudiantes que no tienen estudios previos, mantienen las menores tasas de abandono, pero esta situación podría darse a raíz de estar el estudiante educado en un sistema diferente, pues como menciona (González,1984) la UNED funciona a partir de una metodología novedosa, que busca fundamentalmente eliminar al individuo dependiente y sustituirlo por el autorregulador de su aprendizaje.

Otro elemento influyente en la decisión de continuar o no los estudios es el aspecto laboral, se observa en el primer semestre que el hecho de trabajar no hace diferencia en las tasas de abandono, pero a partir del primer año las diferencias son más marcadas alcanzando mayores tasas el grupo de estudiantes trabajadores, aquí se encuentra una gran contradicción con los objetivos de la creación de la UNED, según González (1984) y Vargas(1996), pues la apertura de esta Universidad iba orientada a la población adulta, laboral y dispersa que por razones obvias no les era factible la asistencia a los centros regulares Universitarios.

En cuanto al lugar de residencia del estudiante, se observa que a lo largo de todo el periodo de estudio las tasas más altas se presentan para los residentes de fuera de la Gran Área Metropolitana, otra aspecto en el que la Universidad Estatal a Distancia de Costa Rica esta fallando, pues fue creada como indica Vargas (1996), accesible a todas las personas que por razones geográficas se les dificultaba el acceso a las otras Universidades Estatales.

Otro factor importante en las tasas de deserción es el número de materias matriculadas, los estudiantes que matriculan 4 materias o más presentan tasas de abandono mayores que el resto de los estudiantes a lo largo del periodo, aquí se presenta un problema de índole administrativo como lo indica Kirkwood (1995), pues tal vez se carece de información sobre 
la carga académica recomendable para un estudiante que posiblemente trabaja, es recomendable que la Universidad establezca un máximo de materias a matricular.

En cuanto a la edad de los estudiantes, los resultados parecen indicar que a mayor edad mayor tasa de abandono, esto podría estar correlacionado con la falta de continuidad en los estudios y como señala Villalobos(1994) esto llevaría a un bajo rendimiento académico y por ende a una posible deserción.

Al analizar la información disponible para poder tratar de descifrar el fenómeno del abandono universitario el objetivo no es solo determinar las causas específicas que llevan al estudiante a abandonar sus estudios, sino también determinar en que medida la institución puede tomar acciones preventivas y no solo ver al abandono como un problema personal del alumno, es posible que la decisión del estudiante se deba a deficiencias del sistema en cuanto a orientación, apoyo y asistencia para los grupos más propensos al abandono, sobre todo en los primeros años de estudio. En general posibilitar realmente los estudios a distancia para aumentar las probabilidades de éxito.

Las características individuales de los estudiantes deben ser tomadas en cuenta a la hora de diseñar los programas o las guías didácticas, pues son condicionantes a sus interrelaciones con su contexto estudiantil y a su integración en la universidad. Sería importante que la universidad tomará en cuenta las características del entorno familiar del estudiante, pues es de esperar que la educación de los padres, el nivel socioeconómico de la familia, el apoyo familiar e inclusive la integración familiar sean elementos importantes que afectan la decisión de abandonar los estudios. Otro elemento importante es el rol que tiene el estudiante dentro de la familia, pues en muchos casos es el sostén económico del hogar.

La UNED debe facilitar información y orientación personalizada al alumno de nuevo ingreso independientemente si tiene estudios previos o no para tratar de disminuir la confusión y el nivel de abandono especialmente en los primeros cursos.

La magnitud del abandono refleja no sólo debilidades en la formación del estudiante que ingresa a la UNED sino también debilidades en el sistema. Por ello se recomienda investigar más profundamente cuáles son las principales razones personales que mueven a un estudiante a tomar esta decisión, pues no basta con admitir estudiantes sino que hay que 
informales y educarlos en este nuevo sistema de enseñanza para lograr en el futuro una relación más coherente entre el número de estudiantes admitidos y el número de estudiantes que culminan con éxito sus estudios.

\section{Referencias}

Barrantes Echavarría, Rodrigo. (1999). Investigación un camino al conocimiento. Un enfoque cuantitativo y cualitativo. San José: Editorial UNED

Barrantes Echavarría, Rodrigo. (1992). Educación a Distancia. San José: Editorial UNED.

Bolaños, Fernando. (1985). Estudios de opinión a un grupo de 61 desertores de la UNED. San José: Editorial UNED.

Carosio, Norma. (1997). Educación a distancia: estrategias para el siglo XXI. En "VIII Congreso Internacional sobre tecnología y educación a distancia" San José: Editorial UNED.

Colás Bravo, Ma Pilar. (1994). Investigación Educativa. Sevilla: Ediciones Alfar, S.A.

Corrales, Maricruz. (1997). Educación a distancia: liderazgo en el siglo XXI. En "VIII Congreso Internacional sobre tecnología y educación a distancia" San José: Editorial UNED.

Curtis, Sian. (1994). Model Further Análisis Plan:Contraceptive Use Dinamics. Life Table Analysis of Discontinuacion, Failure and Switching. New York, Estados Unidos de América: Academic Press.

Cruz Muñoz, Alejandra. (1997). Sociedad y Graduados Universitarios. En "VIII Congreso Internacional sobre tecnología y educación a distancia". San José: Editorial UNED.

Elizondo Solís, Fernando. (1987). La deserción en la educación a distancia, falacias y remedios: una estrategia para enfrentarla. San José: Editorial UNED.

Escotet, Miguel. (1980) Tendencias de la educación superior a distancia. San José: Editorial UNED.

Finardi, Marta. (1997) La educación a distancia frente a los retos educativos del nuevo milenio. En "VIII Congreso Internacional sobre tecnología y educación a distancia". San José: Editorial UNED.

García, Aretio, Lorenzo. (1987) Rendimiento académico y abandono en la educación superior a distancia. Madrid: Editorial UNED

Garland, Maureen R. (1994) Student perceptions of the situational, institutional, dispositional and epistemological barriers to persistence. New York, Estados Unidos de América: Distance Education, 14 (2), 181-198. 
Giovagnoli, Paula Inés. (2002). Determinantes de la deserción y graduación universitaria. Recuperado el 27 de noviembre de 2002 de $\underline{\text { www.depeco.econo.unlp.edu.ar }}$

González, Villegas. (1984). Perspectivas de la Educación a Distancia. San José: Editorial UNED

Granados, Tenorio. (1992). Abandono de Estudios en las Facultades de letras de la UNED. Madrid: Simancas Ediciones S.A.

Hernández Sampieri, Roberto y otros. (1997). Metodología de la Investigación. México D.F.: Editorial McGraw Hill.

Jofré, Arturo. (1998). La universidad en América Latina. Cartago: Editorial Tecnológica de Costa Rica.

Kempfer, Homer. (1996). How to reduce dropouts in distance education. San José: Editorial UNED.

Kirkwood, Adrian. (1995). Evaluación de la Educación a Distancia. New York, Estados Unidos de América: Editorial Wiley.

Namboodiri, K. Y C.M.Suchindran. (1987). Life Table Techniques and Their Applications. New York, Estados Unidos de América: Academic Press.

Otero, Luis. (1983). Deserción un referente conceptual y metodológico para su estudio. San José: Editorial UCR.

Potter, Robert. (1967). The multiple decrement life table as an approach to the measurement of use effectiveness and demographic effectiveness of contraception. Ciudad de Australia: IUSSP. Sydney Conference. Contributed papers.

Ruiz, Ángel. (2001). Educación superior en Costa Rica tendencias y retos en un nuevo escenario histórico. San José: Editorial UCR.

Sanabria, Hernán. (2002). Deserción en estudiantes de Enfermería en cuatro universidades del Perú. Recuperado el 25 de marzo de 2004 de http://sisbid.unmsm.de.pe/bvrevistas/anales/vol63 N4

Teobaldo, Marta. (2004). La deserción de los alumnos universitarios y sus causas. Recuperado el 25 de marzo de 2004 de www.monografías.com/trabajos 10/deserc.shtml

Universidad Estatal a Distancia (UNED). (1994). Boletín de Resúmenes de Publicaciones Sobre Educación a Distancia. № 2-1994. San José: Editorial UNED.

Vargas, Luis Paulino. (1996). UNED de Costa Rica: Realidad y Esperanza. San José: Editorial UNED.

Villalobos, Teresita. (1994). Causas y consecuencias de la deserción estudiantil en la UNA. Tesis de Licenciatura en Orientación del CIDE. Universidad Nacional, Heredia, Costa Rica. 